\title{
Cardiovascular effects of waterpipe smoking: a systematic review and meta-analysis
}

Radwan Al Ali ${ }^{1, *}$, Davor Vukadinović ${ }^{1}$, Wasim Maziak ${ }^{2}$, Lama Katmeh ${ }^{1}$, Viktoria Schwarz ${ }^{1}$, Felix Mahfoud ${ }^{1}$, Ulrich Laufs ${ }^{3}$ and Michael Böhm ${ }^{1}$

${ }^{1}$ Klinik für Innere Medizin III, Kardiologie, Angiologie und Internistische Intensivmedizin, Universitätsklinikum des Saarlandes, Saarland University, Kirrberger Str. 100, 66421 Homburg/Saar, Germany

${ }^{2}$ Robert Stempel College of Public Health, Florida International University, Miami, Florida 33174, USA

${ }^{3}$ Klinik und Poliklinik für Kardiologie, Universitätsklinikum, Liebigstr. 20, 04103 Leipzig, Germany

*Correspondence: radwan.al-ali@uks.eu (Radwan Al Ali)

DOI: $10.31083 /$ i.rcm.2020.03.135

This is an open access article under the CC BY 4.0 license (https://creativecommons.org/licenses/by/4.0/).

Waterpipe smoking has developed into a major and rapidly growing global tobacco epidemic affecting more than 100 million people worldwide. This study identifies and analyzes comprehensively all available data on the cardiovascular effects of waterpipe smoking. Databases PubMed, EMBASE, Web of Science, and the Cochrane Library were searched for studies published until December 2019 assessing cardiovascular effects of waterpipe smoking. We included experimental, cohort, cross-sectional and case-control studies and excluded systematic reviews, case reports/series and qualitative studies. Studies not conducted in humans or not distinguishing waterpipe smoking from other forms of smoking were also excluded. A total of 42 studies with 46 cardiovascular parameters were eligible for analysis. The meta-analysis included 31 studies with 38,037 individuals. Results showed that one waterpipe smoking session leads to immediate increases in heart rate and blood pressure $(P<0.001)$. Compared to non-smokers, waterpipe smokers had significantly lower high-density lipoprotein levels $(P<0.001)$, higher levels of low-density lipoprotein $(P=0.04)$, triglyceride $(P<$ $0.001)$ and fasting blood glucose $(P=0.03)$ and higher heart rate $(P=0.04)$ with a tendency to have higher blood pressure. Mean heart rate, blood pressure and lipids levels did not differ between waterpipe and cigarette smokers, except for total cholesterol, being higher among waterpipe smokers $(P<0.001)$. Current level of evidence suggests that waterpipe smoking is associated with substantial adverse effects on cardiovascular system, which seem to be similar to those of cigarette smoking. Longitudinal studies are required to scrutinize the magnitude of these effects.

\section{Keywords}

Waterpipe; smoking; cardiovascular disease; cardiovascular risk factors

\section{Introduction}

Waterpipe smoking (WPS) has developed into a major and rapidly growing global tobacco epidemic worldwide (Maziak et al., 2015). Several factors have hindered recognition of the harmful effects of WPS especially the flavored smoke and the fashionable aspect. In addition, the water gives the impression that it clears most of harmful substances in the smoke. A single WPS session lasts 30-90 minutes of uninterrupted smoking, producing a large volume of smoke which contains up to 80 times more of toxicants than those which found in the smoke of a single cigarette and carried through the water in the bubbles, so the assumption of users that the smoke is "filtered" by the water is incorrect (ElZaatari et al., 2015; Shihadeh, 2003). Potential adverse cardiovascular effects of WPS have been reported in several scattered studies with varied results based on different estimation criteria. However, the overall clinical effect of WPS on the cardiovascular system is not clear yet. The few available systematic reviews on this topic were not specifically focused on cardiovascular outcomes or synthesis of the results were not performed, being of limited validity (El-Zaatari et al., 2015; Marshall et al., 2018; Rezk-Hanna and Benowitz, 2019; Waziry et al., 2018). We aimed to explore the clinical cardiovascular effects of WPS quantitatively and qualitatively and to compare them with those of cigarette smoking (CS) by combining all available related data in order to provide a better understanding of the relationship between WPS and cardiovascular disease (CVD) risk.

\section{Methods}

\subsection{Study protocol and eligibility criteria}

The analyses were conducted according to a prespecified, nonregistered protocol. A systematic literature search was performed for all published original studies on adults with no limitation on the number of participants. The exposure of interest was WPS and the outcomes of interest were any cardiovascular parameters. We included experimental studies assessing the acute effects of WPS and observational studies (i.e. cohort studies, cross-sectional studies and case-control studies) that compared waterpipe smokers to 
cigarette smokers or non-smokers. Systematic reviews, case reports/case series and qualitative studies were considered not appropriate for inclusion. Furthermore, studies not conducted in humans or not distinguishing WPS from other smoking forms were also excluded (Fig. 1).

\subsection{Search and selection strategy}

Until December 2019, two investigators (R.A., D.V.) independently searched PubMed, EMBASE, Web of Science, and the Cochrane Library using free search text terms combined with Medical Subject Heading (MeSH). The search was performed using variant terms and spellings for waterpipe used in different languages in the title or abstract, or as keywords. Eligibility assessment was performed independently by two investigators (R.A., L.K.). Disagreements were resolved by consulting a third investigator (M.B.).

\subsection{Data extraction}

One investigator extracted the following data from included studies and a second investigator checked the extracted data: 1) study characteristics including study design and settings and sample size; 2) characteristics of study participants including age, gender, health status, smoking status and settings; 3 ) data concerning outcome measurements for each cardiovascular parameter.

\subsection{Risk of bias assessment}

Risk of bias was assessed by two investigators independently (R.A., L.K.) using ROBINS-I (Sterne et al., 2016), a tool recommended from Cochrane Bias Methods Group for assessing the risk of bias in non-randomized interventions. In case of disagreements a third investigator (M.B.) was consulted.

\subsection{Statistical analysis}

Each analysis was conducted by pooling the appropriate data which could be extracted from at least three studies that we considered to be sufficiently similar in their design and comparison groups. Data from experimental studies were used to assess the acute effects of WPS. Combining data from observational studies and baseline data from multigroup experimental studies which have similar control groups were used to assess the non-acute effects of WPS by comparing waterpipe smokers to non- or cigarette smokers. Using a random-effects model, we pooled data from each study including sample size with both average value and standard deviation to estimate pooled mean difference or with prevalence rate to estimate pooled odds ratio. Results for tested parameters were presented using Forest plots along with their respective $95 \%$ confidence interval (CI). Relevant statistical heterogeneity was considered as Cochran's Q test $P<0.05$ and $\mathrm{I}^{2}>50 \%$. As sensitivity analyses, relevant statistical heterogeneity was removed using a previously described "sequential algorithm" approach (Patsopoulos et al., 2008) based on repeating meta-analysis after dropping the outlying study that is responsible for the largest decrease in I2. Funnel plots for parameters reported in $>10$ studies and eventual asymmetry was interpreted as the presence of publication bias. All $P$ values were 2 -sided, with $P<0.05$ considered as significant. All statistical analyses were performed using Review Manager version 5.3 software. We presented summary and descriptive statistics of those parameters, where quantitative (meta-) analysis would not be appropriate.

\section{Results}

\subsection{Search results}

After duplicates were removed, the literature search identified 2,141 studies, of which 149 studies investigated WPS effects. We excluded literature reviews, qualitative studies, case reports/series, and studies conducted among teenagers or on animals or machines. Finally, a total of 42 studies were eligible for inclusion in the systematic review. These included 23 observational (case-control and cross-sectional cohort) studies, which compared waterpipe to nonor cigarette smokers, and 19 experimental studies, all of which were non-randomized and investigated the acute effects of WPS, mostly using one-group pretest-posttest design (Fig. 1). One or more potential longitudinal effects of regular WPS were reported in three of these identified studies (Al Suwaidi et al., 2012; Wolfram et al., 2003; Wu et al., 2013).

\subsection{Study characteristics}

In all experimental studies each parameter was measured at least twice; at start and end of a 15-90-minute WPS session after 12-72-hour smoking abstinence. In the observational studies, waterpipe smokers were in general those who reported regular WPS, mostly twice a week at least. Time since the last WPS session before measurements was rarely reported. Most the included participants were healthy male adults especially in the experimental and case-control studies, except for 7 studies which conducted on patients with coronary heart disease (CHD) (Table. 1). Generally, the search yielded 46 cardiovascular parameters.

\subsection{Statistical analysis and study quality}

Meta-analysis was conducted on 13 parameters including a total of 31 studies with 38037 participants. Most of this analysis showed a significant heterogeneity, which we considered by pooling the data using a random effects model. Based on ROBINS-I criteria we judged 29 studies with moderate and only 2 studies with serious risk of bias. According to the funnel plots there were no signs of publication bias.

\subsection{Study outcomes \\ 3.4.1 Acute effects of waterpipe \\ 3.4.1.1 Hemodynamic parameters \\ 3.4.1.1.1 Heart rate and blood pressure}

To assess the acute effect of WPS on each of heart rate (HR), systolic blood pressure (SBP) and diastolic blood pressure (DBP), meta-analysis was conducted for each of these parameters including 18, 14 and 13 experimental studies with 1814, 1460 and 1386 participants; respectively. Results showed that one WPS session led to acute increases in mean HR by 10.14 beat $/ \mathrm{min}(95 \% \mathrm{CI}$ : 8.41 to $11.88 ; P<0.001$ ), SBP by $7.70 \mathrm{mmHg}$ (95\% CI: 5.13 to 10.27; $P<0.001)$ and DBP by $4.86 \mathrm{mmHg}(95 \% \mathrm{CI}: 2.94$ to 6.78 ; $P<0.001$ ) (Fig. 2). After removing statistical heterogeneity in sensitivity analyses, the acute effect of WPS on these three hemodynamic parameters remained significant.

\subsection{Autonomic regulation}

Potential WPS effects on cardiac autonomic regulation were investigated in three experimental studies (Al-Kubati et al., 2006; Cobb et al., 2012; Nelson et al., 2016), revealing markedly impaired HR/BP variation and baroreflex sensitivity after one WPS session $(P<0.05)$. This effect was independent of nicotine content when tobacco-free-WPS was used (Cobb et al., 2012). 


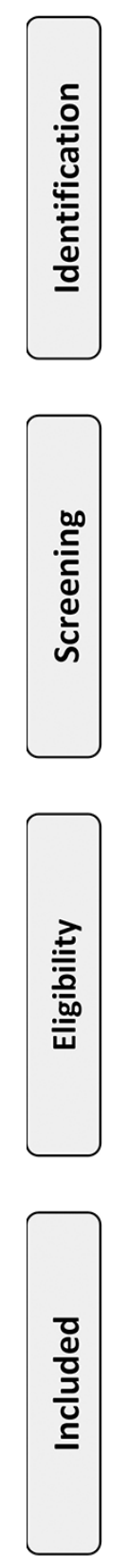

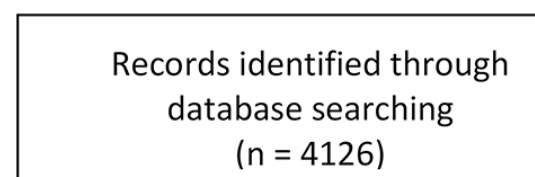

$(n=4126)$
Additional records identified through other sources

$$
(n=0)
$$
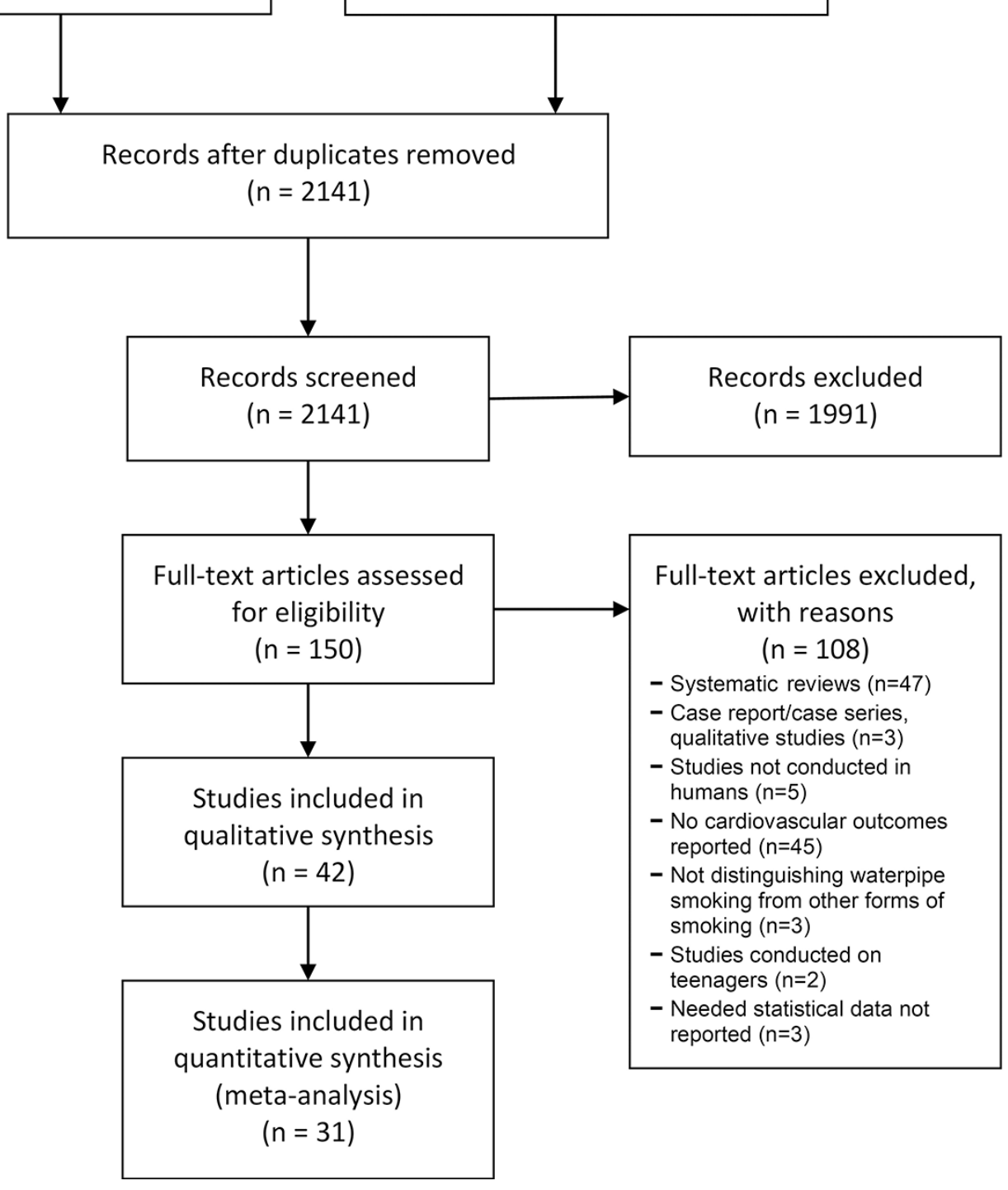

Fig. 1. PRISMA Flow diagram showing the process of the sytematic search and study selection including eligibility against the inclusion and exclusion criteria. The number of studies is the bottom of the flowchart represents that of the selected studies that were considered eligible for inclusion in this meta-analysis. 


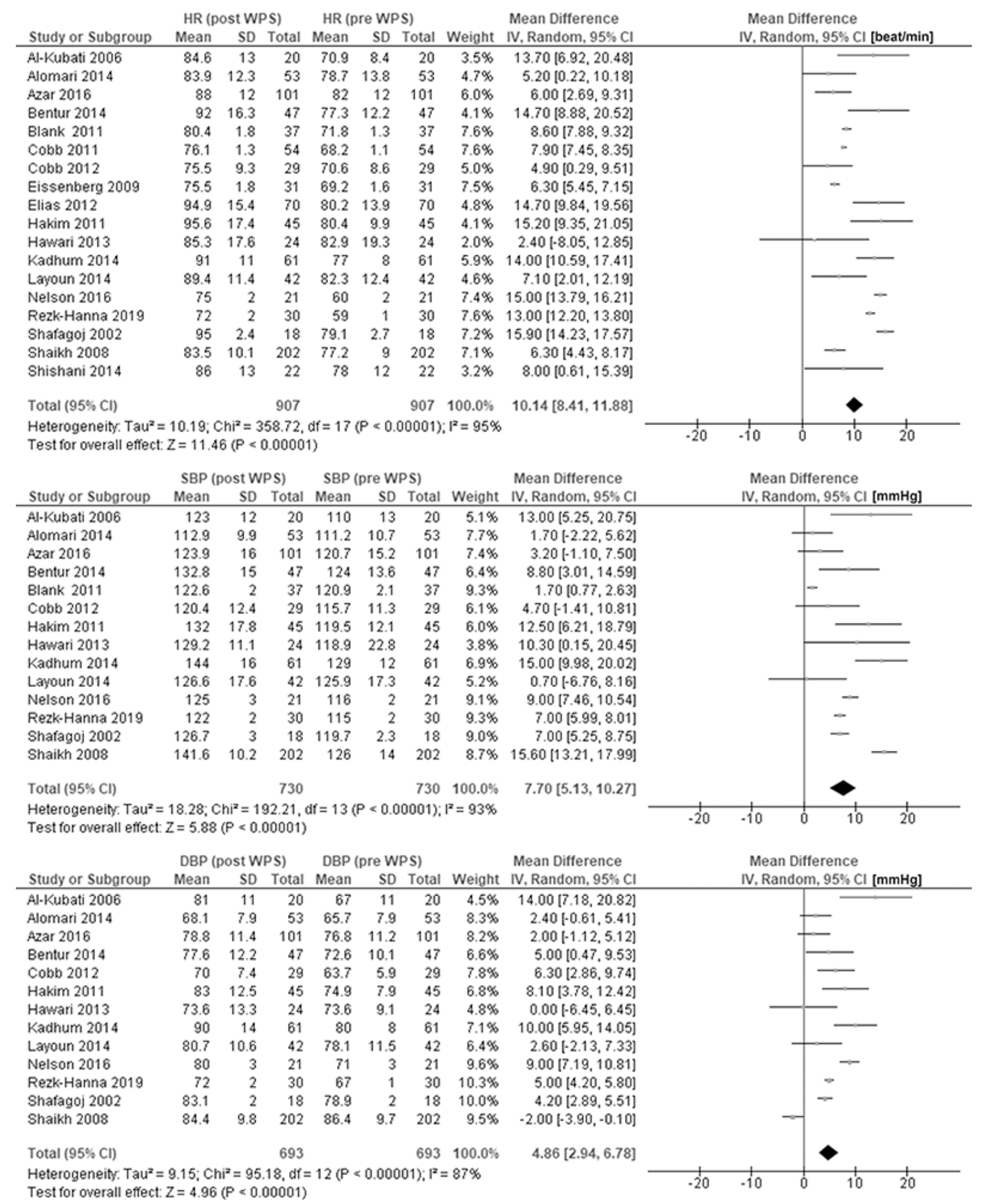

Fig. 2. Forest plots demonstrating individual (squares) and pooled (rhombus) acute changes (mean difference) in heart rate (HR), systolic blood pressure (SBP) and diastolic blood pressure (DBP), with corresponding $95 \%$ confidence intervals (horizontal lines), obtained after one waterpipe smoking session. WPS: Waterpipe smoking 


\begin{tabular}{|c|c|c|c|c|c|c|c|c|c|c|c|c|c|c|c|}
\hline Study & Study design & Sampling & Participants & $\mathbf{N}$ & Male & $\begin{array}{l}\text { Age, years Mean } \\
\pm \text { SD (range) }\end{array}$ & Total & $\begin{array}{r}\text { WPS } \\
\text { Exclusive }\end{array}$ & Frequency & CS & NS & $\begin{array}{l}\text { Pre-session } \\
\text { abstinence }\end{array}$ & $\begin{array}{l}\text { WPS session, } \\
\text { min. }\end{array}$ & Smoking setting & Tobacco used \\
\hline $\begin{array}{l}\text { Shaikh et al., } \\
2008\end{array}$ & $\begin{array}{c}\text { One-Group } \\
\text { Pretest-Posttest }\end{array}$ & Cluster sampling & $\begin{array}{l}\text { Healthy } \\
\text { subjects }\end{array}$ & 202 & 202 & $33.2(>17)$ & 202 & 202 & n.s. & - & - & $20 \mathrm{~min}$ & $30-45$ & Café & n.s. \\
\hline $\begin{array}{l}\text { Shishani et } \\
\text { al., } 2014\end{array}$ & $\begin{array}{c}\text { One-Group } \\
\text { Pretest-Posttest } \\
\text { Two-condition crossover }\end{array}$ & $\begin{array}{l}\text { Voluntary response } \\
\text { sampling }\end{array}$ & $\begin{array}{l}\text { Healthy } \\
\text { subjects }\end{array}$ & 22 & n.s. & $23 \pm 3.1(18-30)$ & 22 & 22 & $\begin{array}{l}\geq 10 \text { times in } \\
\text { the past year, } \\
\text { and } \leq 2 \\
\text { times/week in } \\
\text { the past } 3 \\
\text { months }\end{array}$ & - & - & $24 \mathrm{~h}$ & $45-60$ & $\begin{array}{l}\text { Outdoor } \\
\text { laboratory }\end{array}$ & n.s. \\
\hline $\begin{array}{l}\text { Wolfram et } \\
\text { al., } 2003\end{array}$ & $\begin{array}{c}\text { One-Group } \\
\text { Pretest-Posttest }\end{array}$ & $\begin{array}{l}\text { Convenience } \\
\text { sampling }\end{array}$ & $\begin{array}{l}\text { Healthy } \\
\text { subjects }\end{array}$ & 7 & 7 & $\geq 18$ & 7 & 7 & occasionally & - & - & 3 months & $55(45-70)$ & Laboratory & $\begin{array}{l}15 \mathrm{~g} \text { of } \\
\text { tobacco }\end{array}$ \\
\hline \multicolumn{16}{|c|}{ Case-control studies } \\
\hline $\begin{array}{l}\text { Al-Amri et } \\
\text { al., } 2019\end{array}$ & $\begin{array}{l}\text { Case-control } \\
\text { hospital-based }\end{array}$ & $\begin{array}{l}\text { Convenience } \\
\text { sampling }\end{array}$ & $\begin{array}{l}\text { Cases are } \\
\text { myocardial } \\
\text { infarction, } \\
\text { controls from } \\
\text { dermatology } \\
\text { and surgery } \\
\text { departments }\end{array}$ & 296 & 203 & $47.8 \pm 14.6(\geq 18)$ & 35 & 35 & Daily & 89 & 261 & n.s. & - & - & n.s. \\
\hline $\begin{array}{l}\text { Al-Numair et } \\
\text { al., } 2007\end{array}$ & Case-control & $\begin{array}{l}\text { Convenience } \\
\text { sampling }\end{array}$ & $\begin{array}{l}\text { Healthy } \\
\text { subjects }\end{array}$ & 200 & 200 & $(19-50)$ & 100 & 100 & Daily & - & 100 & n.s. & - & - & ma'ssel \\
\hline $\begin{array}{l}\text { Chami et al., } \\
2019\end{array}$ & $\begin{array}{c}\text { Case-control } \\
\text { community-based }\end{array}$ & $\begin{array}{c}\text { Convenience and } \\
\text { voluntary response } \\
\text { sampling }\end{array}$ & $\begin{array}{l}\text { Healthy } \\
\text { subjects }\end{array}$ & 345 & 233 & $53.7 \pm 9.1(\geq 35)$ & 175 & $98 \%$ & Daily & - & 170 & n.s. & - & - & n.s. \\
\hline $\begin{array}{l}\text { Chwyeed, } \\
2018\end{array}$ & Case-control & Randomly selection & $\begin{array}{l}\text { Healthy } \\
\text { subjects }\end{array}$ & 75 & 75 & $(30-60)$ & 20 & 20 & n.s. & 20 & 35 & n.s. & - & - & n.s. \\
\hline $\begin{array}{l}\text { Diab et al., } \\
2015\end{array}$ & Case-control & $\begin{array}{l}\text { Convenience } \\
\text { sampling }\end{array}$ & $\begin{array}{l}\text { Healthy } \\
\text { subjects }\end{array}$ & 77 & 77 & $35.1 \pm 1.05(\leq 60)$ & 30 & 30 & Daily & 30 & 17 & n.s. & - & - & n.s. \\
\hline $\begin{array}{l}\text { Ghasemi et } \\
\text { al., } 2010\end{array}$ & $\begin{array}{c}\text { Case-control } \\
\text { community-based }\end{array}$ & $\begin{array}{l}\text { Convenience } \\
\text { sampling }\end{array}$ & $\begin{array}{l}\text { Healthy } \\
\text { subjects }\end{array}$ & 54 & 54 & $33.3 \pm 2.94$ & 27 & 27 & Daily & - & 27 & n.s. & - & - & $\begin{array}{c}\text { mostly of } \\
\text { moassal }\end{array}$ \\
\hline $\begin{array}{c}\text { Hashem } \\
\text { Sezavar et } \\
\text { al., } 2004\end{array}$ & $\begin{array}{c}\text { Case-control } \\
\text { community-based }\end{array}$ & $\begin{array}{l}\text { Convenience } \\
\text { sampling }\end{array}$ & n.s. & 450 & 450 & $(20-75)$ & 150 & 150 & Daily & 150 & 150 & n.s. & - & - & n.s. \\
\hline $\begin{array}{l}\text { Jabbour et } \\
\text { al., } 2003\end{array}$ & $\begin{array}{c}\text { Case-control } \\
\text { hospital-based }\end{array}$ & $\begin{array}{l}\text { Convenience } \\
\text { sampling }\end{array}$ & $\begin{array}{l}\text { Cases are CHD } \\
\text { patients, } \\
\text { controls } \\
\text { recruited from } \\
3 \text { hospitals }\end{array}$ & 525 & n.s. & n.s. & 49 & n.s. & $>4 /$ week & - & 299 & n.s. & - & - & n.s. \\
\hline $\begin{array}{l}\text { Koubaa et } \\
\text { al., 2015a }\end{array}$ & $\begin{array}{c}\text { Case-control } \\
\text { community-based }\end{array}$ & $\begin{array}{l}\text { Convenience } \\
\text { sampling }\end{array}$ & $\begin{array}{l}\text { Healthy } \\
\text { subjects }\end{array}$ & 43 & 43 & $43.6 \pm 2.2$ & 14 & 14 & $\geq 5$ WP-year & 15 & 14 & n.s. & - & - & 10 and $25 \mathrm{~g}$ \\
\hline
\end{tabular}




\begin{tabular}{|c|c|c|c|c|c|c|c|c|c|c|c|c|c|c|c|}
\hline Study & Study design & Sampling & Participants & $\mathbf{N}$ & Male & $\begin{array}{l}\text { Age, years Mean } \\
\quad \pm \text { SD (range) }\end{array}$ & Total & $\begin{array}{c}\text { WPS } \\
\text { Exclusive }\end{array}$ & Frequency & CS & NS & $\begin{array}{c}\text { Pre-session } \\
\text { abstinence }\end{array}$ & $\begin{array}{c}\text { WPS } \\
\text { session, mir }\end{array}$ & $\begin{array}{l}\text { Smoking } \\
\text { n. setting }\end{array}$ & $\begin{array}{c}\text { Tobacco } \\
\text { used }\end{array}$ \\
\hline $\begin{array}{l}\text { Koubaa et } \\
\text { al., 2015b }\end{array}$ & $\begin{array}{c}\text { Case-control } \\
\text { community-based }\end{array}$ & $\begin{array}{l}\text { Convenience } \\
\text { sampling }\end{array}$ & Healthy subjects & 43 & 43 & $43.6 \pm 2.2$ & 14 & 14 & $\geq 5$ WP-year & 15 & 14 & n.s. & - & - & 10 and $25 \mathrm{~g}$ \\
\hline $\begin{array}{l}\text { Muddathir et } \\
\text { al., } 2018\end{array}$ & Case-control & $\begin{array}{l}\text { Convenience } \\
\text { sampling }\end{array}$ & Healthy subjects & 120 & 80 & $29.2(18-51)$ & 40 & 40 & Daily & 40 & 40 & n.s. & - & - & n.s. \\
\hline $\begin{array}{l}\text { Selim et al., } \\
\text { 2013a }\end{array}$ & $\begin{array}{c}\text { Case-control } \\
\text { community-based }\end{array}$ & $\begin{array}{l}\text { Convenience } \\
\text { sampling }\end{array}$ & Healthy subjects & 70 & 63 & $28.7(25-35)$ & 30 & 30 & Daily & 30 & 10 & n.s. & - & - & n.s. \\
\hline \multicolumn{16}{|c|}{ Cross-sectional/cohort studies } \\
\hline $\begin{array}{l}\text { Al Suwaidi } \\
\text { et al., } 2012\end{array}$ & $\begin{array}{c}\text { Cross-sectional } \\
\text { prospective } \\
\text { hospital-based cohort }\end{array}$ & $\begin{array}{l}\text { Convenience } \\
\text { sampling }\end{array}$ & ACS patients & 7930 & 6253 & 59.6 & 130 & 130 & Regular & 3605 & 3742 & - & - & - & n.s. \\
\hline $\begin{array}{l}\text { Al-Safi et } \\
\text { al., } 2009\end{array}$ & $\begin{array}{l}\text { Cross-sectional } \\
\text { population-based }\end{array}$ & $\begin{array}{l}\text { Stratified cluster } \\
\text { random sampling }\end{array}$ & Healthy subjects & 14310 & 7400 & $31.4(\geq 18)$ & 2272 & 1132 & $\geq 1 \mathrm{WP} /$ week & 2691 & 9347 & n.s. & - & - & n.s. \\
\hline $\begin{array}{l}\text { Islami et al., } \\
\qquad 2013\end{array}$ & $\begin{array}{c}\text { Cross-sectional } \\
\text { prospective } \\
\text { population-based cohort }\end{array}$ & $\begin{array}{l}\text { Systematic clustering } \\
\text { random sampling }\end{array}$ & $\begin{array}{l}\text { Cases: participants with } \\
\text { heart disease history, } \\
\text { Controls: participants } \\
\text { with no heart disease } \\
\text { history }\end{array}$ & 50045 & 21234 & $(40-75)$ & 525 & n.s. & Ever & - & 49489 & n.s. & - & - & n.s. \\
\hline \multirow[t]{2}{*}{$\begin{array}{c}\text { Khan et al., } \\
2020\end{array}$} & $\begin{array}{l}\text { Cross-sectional } \\
\text { community-based }\end{array}$ & $\begin{array}{l}\text { Voluntary } \\
\text { response sampling }\end{array}$ & Healthy subjects & 73 & 41 & $39.8(21-65)$ & 12 & 12 & Daily & 26 & 25 & n.s. & - & - & n.s. \\
\hline & & & & 57 & 27 & 25.4 & 33 & 33 & $\geq 1 \mathrm{WP} /$ month & & 24 & n.s. & - & - & n.s. \\
\hline $\begin{array}{l}\text { Platt et al., } \\
2017\end{array}$ & $\begin{array}{l}\text { Cross-sectional } \\
\text { hospital-based }\end{array}$ & $\begin{array}{l}\text { Convenience } \\
\text { sampling }\end{array}$ & $\begin{array}{c}\text { Coronary angiography } \\
\text { patients }\end{array}$ & 7705 & 5188 & $61.2 \pm 11.4$ & 574 & 574 & Regularly & 2625 & 4506 & n.s. & - & - & n.s. \\
\hline $\begin{array}{l}\text { Saffar } \\
\text { Soflaei et al., } \\
\quad 2018\end{array}$ & $\begin{array}{l}\text { Cross-sectional } \\
\text { population-based }\end{array}$ & $\begin{array}{l}\text { Stratified cluster } \\
\text { random sampling }\end{array}$ & - & 9690 & n.s. & $(35-65)$ & 1067 & 1067 & n.s. & 864 & 6742 & n.s. & - & - & n.s. \\
\hline $\begin{array}{l}\text { Selim et al., } \\
\text { 2013b }\end{array}$ & $\begin{array}{l}\text { Cross-sectional } \\
\text { hospital-based }\end{array}$ & $\begin{array}{l}\text { Convenience } \\
\text { sampling }\end{array}$ & $\begin{array}{c}\text { Coronary angiography } \\
\text { patients }\end{array}$ & 287 & n.s. & n.s. & 63 & 63 & Regularly & 100 & 109 & n.s. & - & - & n.s. \\
\hline $\begin{array}{l}\text { Shafique et } \\
\text { al., } 2012 \quad \mathrm{p}\end{array}$ & $\begin{array}{c}\text { Cross-sectional } \\
\text { population-based cohort }\end{array}$ & $\begin{array}{l}\text { Voluntary response } \\
\text { sampling }\end{array}$ & Healthy subjects & 2032 & 1039 & $(30-75)$ & 325 & 325 & $\geq 1 \mathrm{WP} /$ week & - & 1707 & n.s. & - & - & n.s. \\
\hline $\begin{array}{l}\text { Sibai et al., } \\
\quad 2014\end{array}$ & $\begin{array}{l}\text { Cross-sectional } \\
\text { hospital-based }\end{array}$ & $\begin{array}{l}\text { Convenience } \\
\text { sampling }\end{array}$ & $\begin{array}{c}\text { Coronary angiography } \\
\text { patients }\end{array}$ & 1754 & n.s. & $(\geq 40)$ & 235 & n.s. & $\begin{array}{l}\text { Ever } \geq 1 \\
\text { WP-year }\end{array}$ & 544 & 975 & n.s. & - & - & n.s. \\
\hline $\begin{array}{l}\text { Ward et al., } \\
\quad 2015\end{array}$ & $\begin{array}{l}\text { Cross-sectional } \\
\text { population-based }\end{array}$ & $\begin{array}{l}\text { Stratified cluster } \\
\text { random sampling }\end{array}$ & - & 2536 & 1220 & $25-65$ & 286 & n.s. & Regularly & - & 2134 & n.s. & - & - & n.s. \\
\hline $\begin{array}{l}\text { Wu et al., } \\
2013\end{array}$ & $\begin{array}{l}\text { Cross-sectional } \\
\text { prospective } \\
\text { population-based cohort }\end{array}$ & $\begin{array}{l}\text { Convenience } \\
\text { sampling }\end{array}$ & n.s. & 20033 & 1971 & $(18-75)$ & n.s. & n.s. & Ever regularly & n.s. & n.s. & n.s. & - & - & n.s. \\
\hline
\end{tabular}

Wु 


\subsubsection{Oxidative status and vascular function \\ 3.4.1.2.1 Oxidative status}

Immediate effects of WPS on endothelial function was investigated through oxidative stress in one study evaluated the acute effect of WPS on the oxidative status (Wolfram et al., 2003), showing a significant increase in mean malondialdehyde from $3.6 \pm 0.4$ to $4.3 \pm 0.3 \mathrm{pg} / \mathrm{ml}(P=0.001), 11$-dehydro-thromboxane-B2 (11DH-TXB2) from $24.9 \pm 1.9$ to $29.8 \pm 2.7 \mathrm{pg} / \mathrm{ml}(P<0.001)$, and 8-Epi-Prostaglandin-F2Alpha (8-epi-PGF2a) from $19.4 \pm 2.4$ to $20.6 \pm 2.6 \mathrm{pg} / \mathrm{ml}(P=0.03)$ after one WPS session.

\subsection{Vascular function}

To investigate the potential acute effect of WPS on vascular function, different clinical methods were used in three experimental (Alomari et al., 2014; Bentur et al., 2014; Rezk-Hanna et al., 2019) and two case-control (Diab et al., 2015; Selim et al., 2013a) studies. One WPS session led to a significantly acute reduction in arterial blood flow by $-8.8 \%(P=0.035)$ and increase in arterial vascular resistance by $16 \%(P=0.003)$ using strain-gauge plethysmography (Alomari et al., 2014), while no acute changes were observed in arterial pulse wave amplitude using "EndoPat" device (Bentur et al., 2014). However, an acute reduction in flow-mediated dilatation (FMD) by $28 \%$ was observed after one WPS session $(P<0.001)$ in a recent study, where the effect of charcoal combustion was removed when the waterpipe-tobaccoproduct was heated electrically (Rezk-Hanna et al., 2019).

\subsubsection{Non-acute effects of waterpipe smoking \\ 3.4.2.1 Hemodynamic parameters \\ 3.4.2.1.1 Heart rate and blood pressure}

Meta-analysis was conducted using data from 10 studies with 14909 participants for HR, 12 studies with 17386 participants for SBP and DBP and 5 studies with 5742 participants for having hypertension. Results showed an increased mean HR by 2.12 (95\% CI: 0.11 to $4.13 ; P=0.04$ ) with a tendency to have higher blood pressure (BP) among waterpipe smokers compared to nonsmokers (Fig. 3). No significant association between WPS and any of these hemodynamic parameters was observed in meta-analysis after eliminating the statistical heterogeneity in sensitivity analyses. However, a large population-based cross-sectional study with 14310 participants (Al-Safi et al., 2009) showed a significant correlation between hemodynamic status and frequency of WPS. Compared to non-smokers, waterpipe smokers of 1-2 sessions/week had higher mean HR by $<1$ beat/min $(P<0.01)$, SBP by $1 \mathrm{mmHg}$ $(P<0.002)$ and DBP by $1 \mathrm{mmHg}(P<0.009)$; and those who smoked $>4$ sessions/week had even higher HR by 3 beat $/ \mathrm{min}$, SBP by $7 \mathrm{mmHg}$ and DBP by $4 \mathrm{mmHg}(P<0.001$ for all comparisons).

\subsubsection{Lipo- and apolipoproteins}

3.4.2.2.1 Lipoproteins

The potential correlation of WPS with serum lipid levels was investigated by conducting the meta-analysis for each of total cholesterol, low-density lipoprotein (LDL)-cholesterol, highdensity lipoprotein (HDL)-cholesterol, triglyceride and having dyslipidemia including 5, 6, 7, 6, and 5 observational studies with 12120, 12320, 14352, 14007 and 13206 participants; respectively. Results demonstrated increased mean LDL-cholesterol by 8.77 $\mathrm{mg} / \mathrm{dl}$ (95\% CI: 0.55 to $17.0 ; P=0.04)$ and triglyceride by 30.6 $\mathrm{mg} / \mathrm{dl}$ (95\% CI: 14.4 to $46.7 ; P<0.001)$ and decreased HDLcholesterol by $-3.39 \mathrm{mg} / \mathrm{dl}$ (95\% CI: -5.13 to $-1.65 ; P<0.001)$ in waterpipe smokers compared to non-smokers (Fig. 4). The correlation between WPS and increased triglyceride and decreased HDL-cholesterol remained significant after removing statistical heterogeneity in sensitivity analyses.

\subsection{Apolipoproteins}

One case-control study (Al-Numair et al., 2007) compared apolipoproteins (Apo) between waterpipe smokers and nonsmokers, revealing decreased mean Apo-A1 (46 \pm 1.10 vs. $42 \pm$ $1.92 \mathrm{mmol} / \mathrm{l} ; P<0.05)$ and increased mean Apo-B (2.02 \pm 0.61 vs. $2.38 \pm 0.40 ; P<0.05)$ among waterpipe smokers.

\subsubsection{Coagulation factors} 3.4.2.3.1 Fibrinogen

Three case-control studies investigated the association between WPS and fibrinogen (Hashem Sezavar et al., 2004; Khan et al., 2020; Muddathir et al., 2018), where significantly increased levels of fibrinogen by about $15-25 \%$ were observed among waterpipe smokers compared to non-smokers $(P<0.01)$ in two of them (Hashem Sezavar et al., 2004; Muddathir et al., 2018).

\subsection{Factor-VII and VIII}

Factor-VII and VIII were investigated in one study (Muddathir et al., 2018), showing higher levels by about $25 \%$ and $50 \%$, respectively, in waterpipe smokers than in non-smokers, especially in those who smoked waterpipe for more than 3 years $(P<0.01)$.

\subsection{Other coagulation factors}

No significant long-term effect has been reported on Serpine1/Plasminogen activator inhibitor-1 (Khan et al., 2020).

\subsubsection{Oxidative status, inflammation, and vascular function \\ 3.4.2.4.1. Oxidative status and inflammation}

One study investigated the longitudinal effect of WPS on the oxidative status (Wolfram et al., 2003). Blood levels of malondialdehyde, 11-DH-TXB2 and 8-epi-PGF2a continued to rise over two weeks of daily WPS and remained significantly elevated even before the initiation of a new WPS session.

Two (Al-Numair et al., 2007; Koubaa et al., 2015a) of three studies (Al-Numair et al., 2007; Khan et al., 2020; Koubaa et al., 2015a) observed significantly increased levels of malondialdehyde among waterpipe smokers compared to non-smokers. These two studies also showed significantly reduced levels of total antioxidant capacity (TAC) among waterpipe smokers compared to nonsmokers $(P<0.05)$. No significant differences were found regarding plasma myeloperoxidase and Intercellular Adhesion Molecule1. However, urinary levels of 8-isoprostanes and myeloperoxidase were significantly higher in waterpipe smokers than in nonsmokers (Khan et al., 2020; Koubaa et al., 2015a).

In one study, Nitric oxide (NO) metabolites were measured in waterpipe smokers (Ghasemi et al., 2010) demonstrating higher levels than in non-smokers ( 34.3 vs. 22.5 micromol/1; $P<0.01$ ). No difference was observed regarding $C$-reactive protein (CRP) in three studies (Diab et al., 2015; Khan et al., 2020; Saffar Soflaei et al., 2018). 


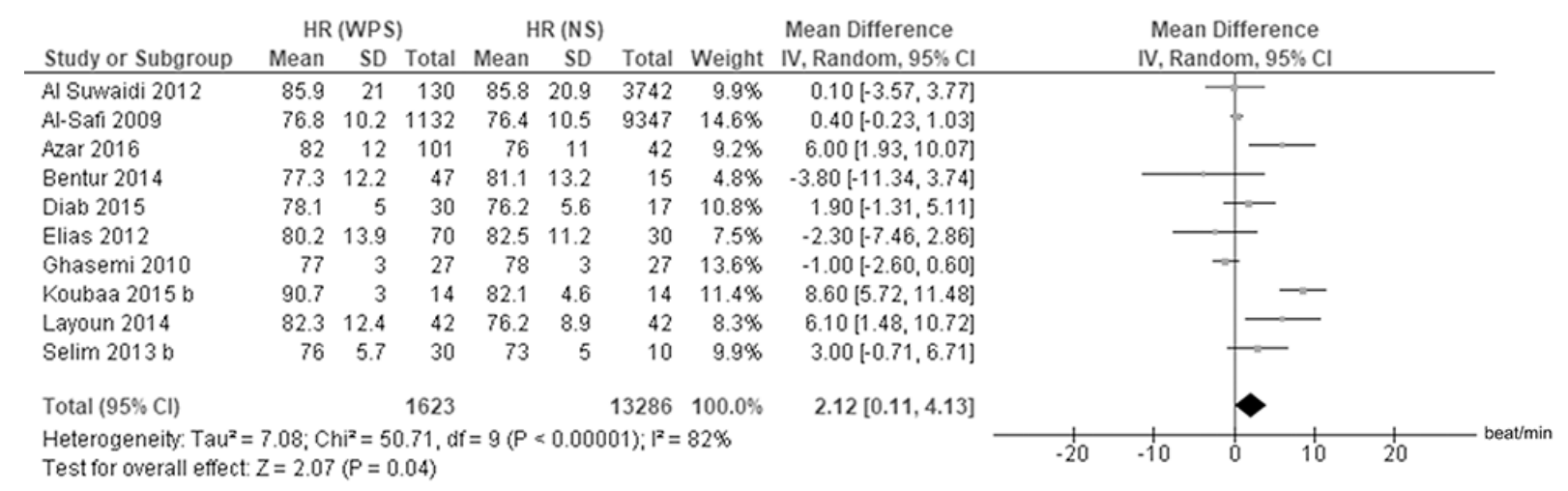

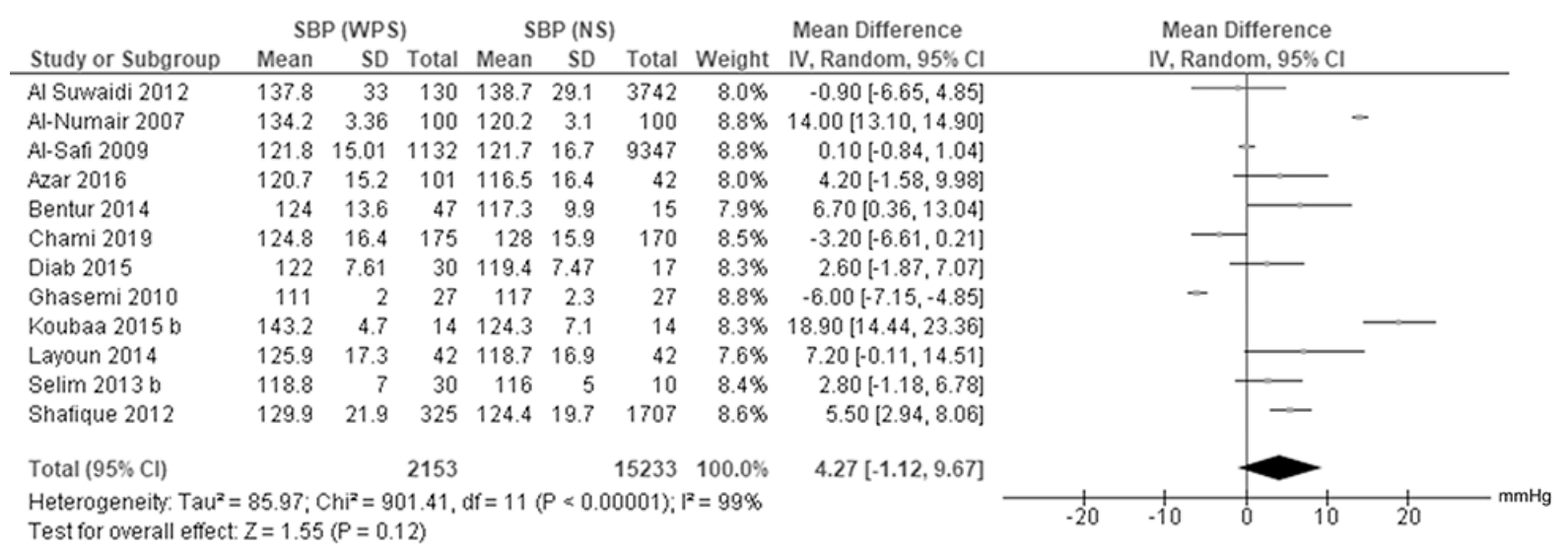

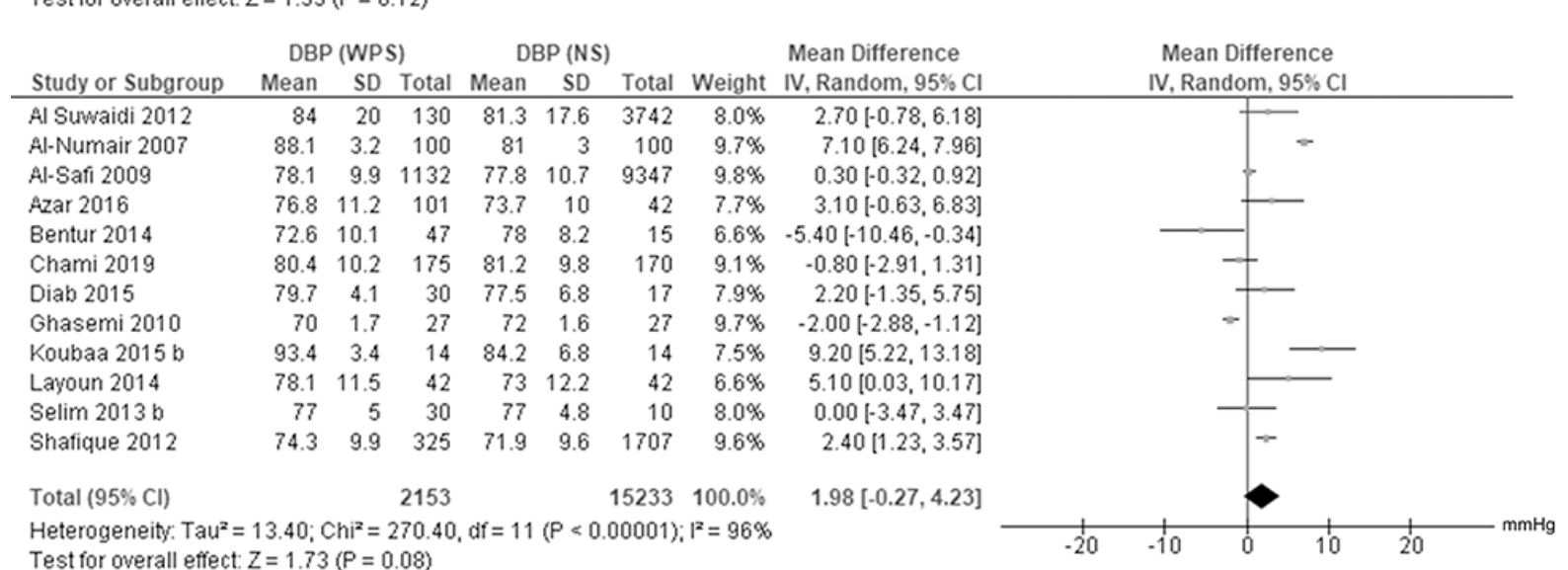

Fig. 3. Forest plots demonstrating individual (squares) and pooled (rhombus) mean differences in heart rate (HR), systolic blood pressure (SBP) and diastolic blood pressure (DBP), with corresponding $95 \%$ confidence intervals (horizontal lines), obtained in waterpipe smokers compared to non-smokers. WPS: Waterpipe smoking. NS: Non-smoking.

\subsection{Vascular function}

Two case-control studies evaluated the potential non-acute effect of regular WPS on FMD, where lower FMD values by about $30-60 \%$ were observed in waterpipe smokers than in non-smokers $(P<0.05)$ (Diab et al., 2015; Selim et al., 2013a), with a negative correlation between FMD and both smoking duration in years $(P$ $<0.001)$ and number of daily sessions $(P<0.001)$ (Selim et al., 2013a).

\subsubsection{Fasting blood glucose}

Our meta-analysis showed that waterpipe smokers had a higher mean fasting blood glucose (FBG) of $4.66 \mathrm{mg} / \mathrm{dl}$ (95\% CI: 0.53 to $8.80 ; P=0.03)$ than non-smokers did, with no association between
WPS and having diabetes mellitus (DM). After removing the statistical heterogeneity in sensitivity analyses, a significant correlation was revealed between WPS and both of DM $(\mathrm{OR}=1.35 ; 95 \%$ CI: 1.16 to $1.57 ; P<0.001)$ and mean FBG.

\subsubsection{Body mass index}

No difference was observed between waterpipe smokers and non-smokers regarding body mass index (BMI) in our metaanalysis. However, two population-based cross-sectional studies observed a significant association between obesity and each of regular waterpipe smokers $(\mathrm{OR}=1.44 ; 95 \% \mathrm{CI}$ : 1.26 to $1.65 ; P$ $<0.001$ ) (Saffar Soflaei et al., 2018) and daily waterpipe smokers $(\mathrm{OR}=2.87 ; 95 \% \mathrm{CI}: 1.06$ to $7.76 ; P=0.038)$ (Ward et al., 


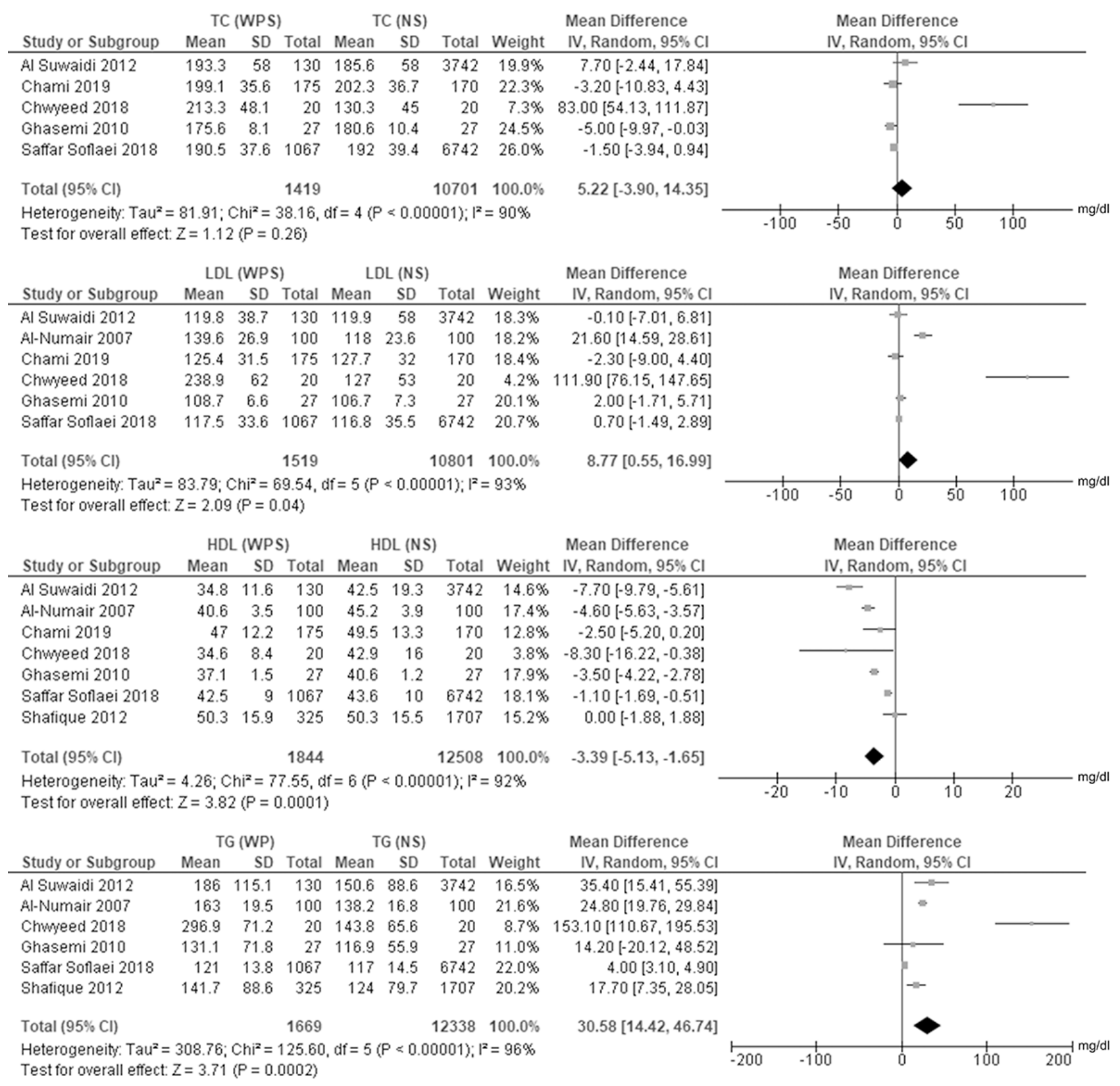

Fig. 4. Forest plots demonstrating individual (squares) and pooled (rhombus) mean differences in blood levels of total cholesterol (TC), lowdensity lipoprotein cholesterol (LDL), high-density lipoprotein cholesterol (HDL) and triglyceride (TG), with corresponding 95\% confidence intervals (horizontal lines), obtained in waterpipe smokers compared to non-smokers. WPS: Waterpipe smoking. NS: Non-smoking.

2015), but no significant correlation was observed in a hospital based cross-sectional study (Selim et al., 2013b).

\subsubsection{Metabolic syndrome and 10-year CHD risk}

Two population-based studies (Saffar Soflaei et al., 2018; Shafique et al., 2012) assessed the potential correlation of WPS with metabolic syndrome (MS). Waterpipe smokers were more likely to have MS compared to non-smokers ( $\mathrm{OR}=1.29 ; 95 \% \mathrm{CI}$ : 1.12 to $1.48 ; P<0.001$; and $\mathrm{OR}=3.21 ; 95 \%$ CI: 2.38 to $4.33 ; P$ $<0.01)$.

One case-control study (Chami et al., 2019) evaluated the 10year CHD risk in waterpipe smokers, showing higher mean risk score in waterpipe smokers than in non-smokers $(7.12 \pm 7.18$ vs. $4.05 \pm 4.48 ; P<0.001)$.

\subsubsection{Coronary artery calcium}

Coronary Artery Calcium (CAC) score was measured among waterpipe smokers in one case-control study (Chami et al., 2019). Waterpipe smokers had higher mean CAC score than non-smokers $\operatorname{did}(90.6 \pm 400.3$ vs. $52.8 \pm 218.6$ AUs; $P=0.02)$.

\subsubsection{Cardiovascular disease \\ 3.4.2.9.1 Incidence of cardiovascular disease}

A significant correlation between WPS and the incidence of CVD was reported in four out of seven differently designed stud- 
ies (Al-Amri et al., 2019; Al Suwaidi et al., 2012; Chami et al., 2019; Islami et al., 2013; Jabbour et al., 2003; Platt et al., 2017; Saffar Soflaei et al., 2018). In a large population-based cohort study with 50045 participants (Islami et al., 2013) heart disease correlated to the consumption of $>180$ waterpipe-year (OR: 3.75 ; $95 \%$ CI: 1.52 to 9.22 ). A recent hospital-based case-control study showed that myocardial infarction (MI) was independently associated with WPS (OR = 10.3; 95\% CI: 2.22 to 47.29) (Al-Amri et al., 2019). Similarly, WPS significantly associated with having MI in a cross-sectional hospital-based on 7705 patients referred for cardiac catheterization $(\mathrm{OR}=1.3 ; 95 \% \mathrm{CI}: 1.04$ to $1.68 ; P=0.021)$ (Platt et al., 2017) and with having ST-elevation MI (STEMI) in another cross-sectional hospital-based on 7930 patients admitted with acute coronary syndrome (ACS) (Al Suwaidi et al., 2012).

\subsection{Severity of coronary artery disease}

Two cross-sectional studies assessed the correlation between WPS and the severity of coronary artery disease (CAD). Compared to non-smokers, waterpipe smokers had a higher mean Duke Jeopardy score (DJS) of anatomical extension of CAD $(P<0.05)$, with a positive correlation between DJS and the duration of smoking in years $(\mathrm{r}=0.574, P<0.001)$ (Selim et al., 2013b). Waterpipe smokers with $>40$ waterpipe-years were more likely to have severe coronary stenosis $(\mathrm{OR}=2.94 ; 95 \% \mathrm{CI}$ : 1.04-8.33) with a higher Duke CAD prognostic index $(\beta=7.835, P=0.027)$ than non-smokers did (Sibai et al., 2014).

\subsection{Outcomes of cardiovascular disease}

The relationship between WPS and CVD outcomes was investigated in two cross-sectional studies (Al Suwaidi et al., 2012; Wu et al., 2013). Among patients with ACS, waterpipe smokers were more likely to develop arrhythmias at presentation $(\mathrm{OR}=2.0 ; 95 \%$ CI: 1.08 to $3.69 ; P=0.026)$ and in-hospital complications (OR = 2.7; CI 95\%: 1.85 to $3.88, P<0.001$ ), especially recurrent myocardial ischemia (OR $=2.08 ; 95 \%$ CI: 1.40 to $3.10 ; P<0.001)$, than non-smokers did. No significant differences were observed regarding Killip classification or mortality rate (Al Suwaidi et al., 2012). However, in a large prospective population-based cohort study with 20033 participants, WPS for $>5$ times/day significantly correlated with increased risk of death from ischemic heart disease (HR $=1.96 ; 95 \% \mathrm{CI}: 1.05$ to $3.63 ; P=0.04)$ (Wu et al., 2013).

\subsubsection{Waterpipe compared with cigarette smoking 3.4.3.1 Heart rate and blood pressure}

Meta-analysis including data from seven observational studies with 7842 participants showed no differences between waterpipe and cigarette smokers regarding HR, SBP or DBP (Fig. 5). After removing the statistical heterogeneity in sensitivity analyses, significantly increased mean HR by 3.21 (95\% CI: 2.31 to 4.11 ; $P<0.001$ ), SBP by 2.82 (95\% CI: 1.15 to, $4.49 ; P=0.001)$ and DBP by 2.38 (95\% CI: 0.98 to $3.79 ; P<0.001$ ) were revealed. One of two studies (Al Suwaidi et al., 2012; Selim et al., 2013b) observed higher hypertension rates $(53.1 \%$ vs. $39.2 \% ; P=0.001)$ (Al Suwaidi et al., 2012) in waterpipe than in cigarette smokers.

\subsubsection{Lipoproteins}

Pooled data from three observational studies with 5721 participants showed higher mean total cholesterol of $6.80 \mathrm{mg} / \mathrm{dl}$ (95\% CI: 3.23 to $10.38 ; P<0.001)$ in waterpipe than in cigarette smokers. No differences were found regarding levels of LDL-cholesterol, HDL-cholesterol or triglyceride or having dyslipidemia (Fig. 6). However, a significant correlation between WPS and triglyceride level was revealed after removing the statistical heterogeneity in sensitivity analyses.

\subsubsection{Body mass indeex and diabetes mellitus}

Meta-analysis showed no differences regarding mean BMI and prevalence of DM between waterpipe and cigarette smokers. However, one of two studies (Saffar Soflaei et al., 2018; Selim et al., 2013b) reprted a higher obesity rat (OR $=4.19 ; 95 \%$ CI: 3.33 to 5.28; $\mathrm{P}<0.001$ ) in waterpipe than in cigarette smokers (Saffar Soflaei et al., 2018).

\subsubsection{Oxidative status, inflammation, and vascular function}

Meta-analysis showed no difference regarding FMD between waterpipe and cigarette smokers. Furthermore, the three studies that compared CRP levels (Diab et al., 2015; Khan et al., 2020; Saffar Soflaei et al., 2018) and the two studies that compared levels of malondialdehyde and TAC (Khan et al., 2020; Koubaa et al., 2015a) observed no differences between waterpipe and cigarette smokers.

\subsubsection{Coagulation factors}

In two of the three case-control studies (Hashem Sezavar et al., 2004; Khan et al., 2020; Muddathir et al., 2018), waterpipe smokers had significantly higher levels of fibrinogen than cigarette smokers did $(P<0.05)$ (Hashem Sezavar et al., 2004; Muddathir et al., 2018). The one study which investigated the levels of factorVII and VIII (Muddathir et al., 2018) found no significant difference between waterpipe and cigarette smokers.

\subsubsection{Cardiovascular Disease}

Different aspects of CVD were compared between waterpipe and cigarette smokers in three studies (Al Suwaidi et al., 2012; Saffar Soflaei et al., 2018; Selim et al., 2013b). No differences were observed regarding CVD incidence (Al Suwaidi et al., 2012; Saffar Soflaei et al., 2018). However, higher mean DJS was also observed in waterpipe than in cigarette smokers in a cross-sectional study conducted on CAD patients $(P<0.05)$ (Selim et al., 2013b). In another study on ACS patients, waterpipe smokers were more likely to have arrhythmia at presentation $(\mathrm{OR}=1.79 ; 95 \% \mathrm{CI}$ : 1.26 to $2.54 ; P=0.001$ ), in-hospital cardiovascular complications (OR $=3.37 ; 95 \%$ CI: 2.33 to $4.89 ; P<0.001$ ), especially recurrent ischemia (OR=2.25; $95 \% \mathrm{CI}$ : 1.51 to $3.35 ; P<0.001)$, higher mortality rate $(\mathrm{OR}=1.8 ; 95 \% \mathrm{CI}: 1.06$ to $3.09 ; P<0.05)$ and killip classification of $>1(\mathrm{OR}=1.7 ; 95 \% \mathrm{CI}: 1.14$ to $2.45 ; P<0.01)$ than cigarette smokers did (Al Suwaidi et al., 2012).

\section{Discussion}

The main findings of our analyses are: i) WPS leads to an acute increase in HR, SBP and DBP; ii) waterpipe smokers have increased HR, higher triglyceride and LDL-Cholesterol and lower 


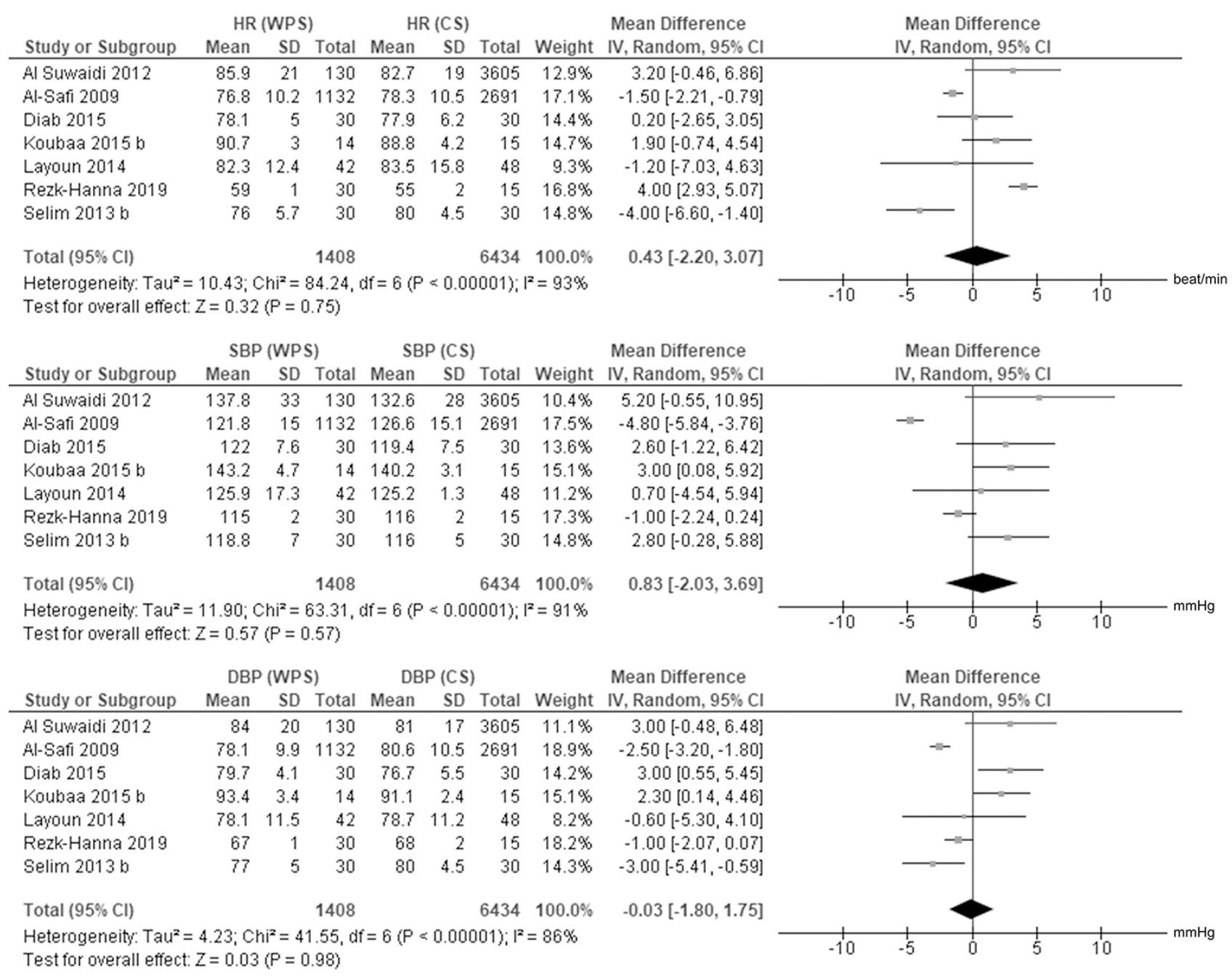

Fig. 5. Forest plots demonstrating individual (squares) and pooled (rhombus) mean differences in heart rate (HR), systolic blood pressure (SBP) and diastolic blood pressure (DBP), with corresponding $95 \%$ confidence intervals (horizontal lines), obtained in waterpipe smokers compared to cigarette smokers. WPS: Waterpipe smoking. CS: Cigarette smoking.

HDL-Cholesterol levels compared to non-smokers; iii) the cardiometabolic profile in waterpipe smokers is not less worse than in cigarette smokers.

It is well known that increased $\mathrm{HR}$ and $\mathrm{BP}$, the two most widely used hemodynamic parameters in assessment of cardiovascular system, negatively affect cardiovascular outcome. (Ettehad et al., 2016; Nikolovska Vukadinović et al., 2017). According to our results one WPS session causes acute increase in HR by about 11 beats/minute, SBP by $7 \mathrm{mmHg}$ und DBP by $5 \mathrm{mmHg}$. This itself may lead to increased oxygen demand of the heart, augment shear stress of the blood vessel, which in some cases may provoke ACS, thereby increasing morbidity and mortality. Based on available data, it is not known how long these acute hemodynamic effects of WPS might last. The answer to this question needs more studies with serial measurements of these parameters. As waterpipe is mostly consumed regularly for several times a week, it could be expected that accumulation of these acute adverse effects negatively impacts prognosis over time. However, our results show that waterpipe smokers had slightly increased HR in comparison with non-smokers for about 2 beats/minute, while SBD and DBP tend to be higher among waterpipe smokers but without reaching the statistical significance. These results differ somewhat unexpectedly from those observed with the acute effects of WPS, which could be partially explained through large heterogeneity across the studies. Furthermore, frequency and duration of WPS sessions and years of smoking were not adjusted across the studies, which might affect the results. A significant positive correlation was previously observed between the number of weekly use of waterpipe and each of SBP, SBP and HR (Al-Safi et al., 2009). The acute hemodynamic changes revealed in our analyses may be attributed to some extent to nicotine exposure, which augments the sympathetic nervous system activity, leading to increases in HR, myocardial contractility and cardiac output (Salahuddin et al., 2012). Such an effect has been reported in the three cross-over design studies (Blank et al., 2011; Cobb et al., 2012; Shishani et al., 2014) comparing flavor-matched tobacco- with tobacco-free-WPS. However, an acute cardiac autonomic dysregulation was observed after a WPS session independently of nicotine content (Cobb et al., 2012). In 


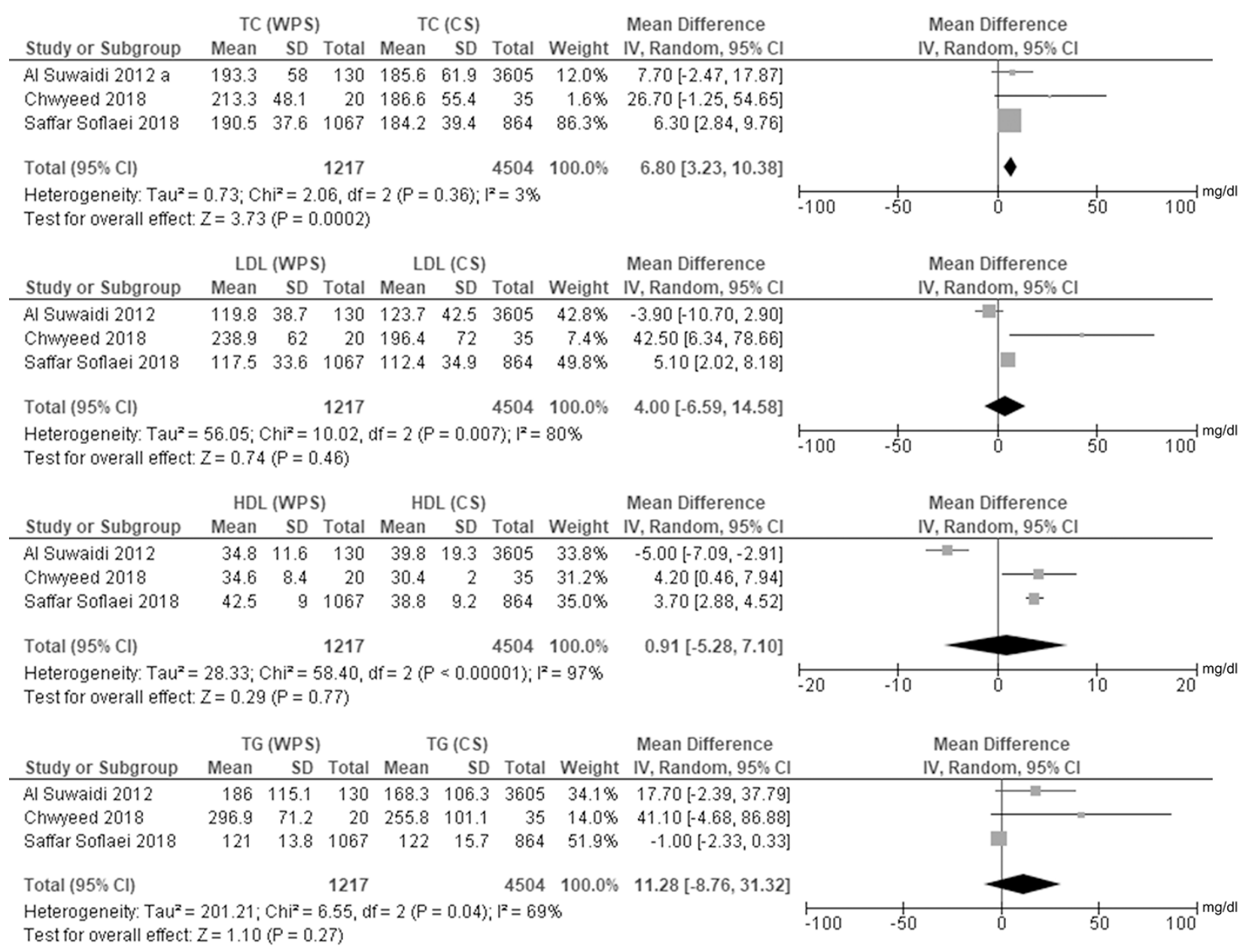

Fig. 6. Forest plots demonstrating individual (squares) and pooled (rhombus) mean differences in blood levels of total cholesterol (TC), lowdensity lipoprotein cholesterol (LDL), high-density lipoprotein cholesterol (HDL) and triglyceride (TG), with corresponding 95\% confidence intervals (horizontal lines), obtained in waterpipe smokers compared to cigarette smokers. WPS: Waterpipe smoking. CS: Cigarette smoking.

addition, the high levels of exposed CO during WPS (Eissenberg and Shihadeh, 2009) can lead to decreased oxygen supply to tissues including the heart due to the formation of $\mathrm{CO}-\mathrm{Hb}$ (Benowitz, 2003). In turn, it has been well established that hypoxia is a potent stimulator of several autonomic mechanisms leading to increases in resting HR, BP and cardiac output (Vigo et al., 2010). These findings contradict harm reduction claims of so-called "herbal" waterpipe-products and correspond to outcomes of non-clinical studies on such products using a human-mimic waterpipe-machine (Hammal et al., 2015). Owing to the lack of data from longitudinal studies, it is not possible to determine to what extent WPS can be hemodynamically harmful at the long-term.

Pooling data from available studies revealed a significant correlation between WPS and increased triglyceride and LDLcholesterol and decreased HDL-cholesterol, which are recognized as CVD risk factors that promote atherosclerosis (Pedro-Botet et al., 2020). As is well known for CS, the mechanisms responsible are not clearly elucidated. However, the triglyceride/high-density lipoprotein abnormalities have recently been suggested to be related to insulin resistance. (Ambrose and Barua, 2004). This can be supported with our results that showed a significant increase in
FBG in waterpipe smokers compared to non-smokers.

The association between CS and increased activity of coagulation factors and thrombotic risk has been previously reported (Eliasson, 1995). Likewise, WPS also correlates with increased levels of fibrinogen and factors VII and VIII (Hashem Sezavar et al., 2004; Khan et al., 2020; Muddathir et al., 2018), which may increase thrombogenicity, thus increasing the risk for cardiovascular events. The harmful effects of WPS are reflected by the increased CAC score (Chami et al., 2019) and the acute (Alomari et al., 2014; Rezk-Hanna et al., 2019; Wolfram et al., 2003) and long-term (AlNumair et al., 2007; Diab et al., 2015; Ghasemi et al., 2010; Koubaa et al., 2015a; Selim et al., 2013a) endothelial dysfunction, which were established among waterpipe smokers, providing clinical evidence for the potential contribution of WPS to vascular disease. Our findings on WPS effects on cardiovascular system explain and support results of studies which reported a significant correlation between WPS and each of incidence (Al-Amri et al., 2019; Islami et al., 2013; Jabbour et al., 2003; Platt et al., 2017), worse clinical outcomes (Al Suwaidi et al., 2012) and estimated prognoses (Selim et al., 2013b; Sibai et al., 2014) of CVD.

As cardiovascular effects of CS are well known (Ambrose and 
Barua, 2004; Leone, 2003; Mons et al., 2015), the comparison between WPS and CS is of a great importance. Unfortunately, fewer studies could be included for this comparison. The lack of studies reporting the rate of cardiovascular and cerebrovascular events in waterpipe vs cigarette smokers may be the main limitation. However, based on our results, the non-acute effects of WPS on the vast majority of cardiovascular parameters of interest seem similar to those produced by CS. The few available studies showed no clear difference in the CVD incidence between WPS and CS (Al Suwaidi et al., 2012; Saffar Soflaei et al., 2018). Furhtermore, CVD complications (Al Suwaidi et al., 2012) and mortality (Al Suwaidi et al., 2012) tend to be of higher rates in waterpipe than in cigarette smokers. This might be explained due to prolonged WPS and, therefore, cumulatively higher amounts of inhaled toxic substances and consequently adverse effects on the cardiovascular system (Sibai et al., 2014).

\section{Strengths and limitations}

A limitation of these analyses is the high heterogeneity of the available data. This can be attributed to the geographic diversity of the analyzed studies, the multiple study protocols, as well as different frequency and session duration of WPS. Furthermore, some parameters were reported in only few studies, which limits the explanatory power of this analysis. Therefore, some results should be interpreted with caution. As long-term follow-up studies on cardiovascular effects of WPS are scarce, chronic effect of WPS has been estimated in our analyses using data from available case-control and cross-sectional cohort studies that compared waterpipe smokers to non-smokers. Of note, the observed effects could be explained solely due to WPS, as limited number of waterpipe smokers may occasionally have also smoked cigarettes. Although the comparison between WPS and CS may be the most important part of systematic review, only few studies are available for this comparison, with the main limitation is the lack of studies reporting the rate of cardiovascular and cerebrovascular events. On the other hand, some waterpipe smokers could be ex-cigarette smokers. The time spent smoking cigarette are likely impacting, observed outcomes. Such information is missed in most observational studies. Thus, a meta-regression considering time from quitting of cigarette smoking in waterpipe smokers is not possible. This applies to the other results showed a worst cardiometabolic profile of waterpipe smokers compared to non-smokers, as many studies did not consider all potential confounders in the comparisons. To the best of our knowledge, our study provides the most comprehensive image of the potential cardiovascular effects of WPS based on scientific evidence and reflects the current magnitude of research efforts performed so far regarding this issue.

\section{Conclusions}

There is still a widespread believe that WPS is harmless and not real smoking. This wide-ranged systematic review and metaanalysis outlines the spectrum of acute and long-term cardiovascular effects of waterpipe smoking. Despite all the stated limitations, current level of evidence suggests that WPS is associated with substantial adverse effects on cardiovascular system, which seem to be similar to what reported for cigarette smoking. Further research is required especially longitudinal studies evaluating the long-term consequences of both waterpipe smoking and cessation to scrutinize the magnitude of these effects and to provide a strong evidence for a causal relationship.

\section{Authors' contributions}

Concept and design: RA and MB. Literature search for eligible studies: RA, DV, and LK. Data extraction and quality assessment RA and LK. Data analysis and interpretation: RA, MB and DV. Writing of original draft: RA. Review and editing of article: all authors. Critical revision of article: RA, MB and DV. Approval of article: all authors.

\section{Acknowledgments}

The authors thank all the peer reviewers and editors for their opinions and suggestions.

\section{Conflict of interest}

Authors do not have any conflict of interest related to this study.

Submitted: July 14, 2020

Revised: September 09, 2020

Accepted: September 12, 2020

Published: September 30, 2020

\section{References}

Al-Amri, A., Ghalilah, K., Al-Harbi, A., Al-Dubai, S. A. R., Al-Ghamdi, S. and Al-Zalabani, A. (2019) Waterpipe smoking and the risk of myocardial infarction: a hospital-based case-control study. Tobacco Induced Diseases 17, 87-87.

Al-Kubati, M., Al-Kubati, A. S., al'Absi, M. and Fišer, B. (2006) The short-term effect of water-pipe smoking on the baroreflex control of heart rate in normotensives. Autonomic Neuroscience 126-127, 146149 .

Al-Numair, K., Barber-Heidal, K., Al-Assaf, A. and El-Desoky, G. (2007) Water-pipe (shisha) smoking influences total antioxidant capacity and oxidative stress of healthy saudi males. Journal of Food Agriculture and Environment 5, 17-22.

Al-Safi, S. A., Ayoub, N. M., Albalas, M. A., Al-Doghim, I. and AboulEnein, F. H. (2009) Does shisha smoking affect blood pressure and heart rate? Journal of Public Health 17, 121-126.

Al Suwaidi, J., Al Habib, K., Singh, R., Hersi, A., Al Nemer, K., Asaad, N., Al Saif, S., Al-Motarreb, A., Almahmeed, W., Sulaiman, K., Amin, H., Al-Lawati, J., Al Bustani, N., Al-Sagheer, N. Q. and Ali, W. M. (2012) Tobacco modalities used and outcome in patients with acute coronary syndrome: an observational report. Postgraduate Medical Journal $\mathbf{8 8}$ 566-574.

Alomari, M. A., Khabour, O. F., Alzoubi, K. H., Shqair, D. M. and Eissenberg, T. (2014) Central and peripheral cardiovascular changes immediately after waterpipe smoking. Inhalation Toxicology 26, 579-587.

Ambrose, J. A. and Barua, R. S. (2004) The pathophysiology of cigarette smoking and cardiovascular disease. Journal of the American College of Cardiology 43, 1731-1737.

Azar, R. R., Frangieh, A. H., Mroué, J., Bassila, L., Kasty, M., Hage, G. and Kadri, Z. (2016) Acute effects of waterpipe smoking on blood pressure and heart rate: a real-life trial. Inhalation Toxicology 28, 339-342.

Benowitz, N. L. (2003) Cigarette smoking and cardiovascular disease: pathophysiology and implications for treatment. Progress in Cardiovascular Diseases 46, 91-111.

Bentur, L., Hellou, E., Goldbart, A., Pillar, G., Monovich, E., Salameh, M., Scherb, I. and Bentur, Y. (2014) Laboratory and clinical acute effects of active and passive indoor group water-pipe (narghile) smoking. Chest 145, 803-809.

Blank, M. D., Cobb, C. O., Kilgalen, B., Austin, J., Weaver, M. F., Shi- 
hadeh, A. and Eissenberg, T. (2011) Acute effects of waterpipe tobacco smoking: a double-blind, placebo-control study. Drug and Alcohol Dependence 116, 102-109.

Chami, H. A., Isma'eel, H., Tamim, H., Adawi, M., Al Kuwari, M. and Al Mullah, A. (2019) The association of water-pipe smoking and coronary artery calcium in a community-based sample. Chest 155, 1217-1225.

Chwyeed, S. (2018) A Comparison between the Effect of Shisha and Cigarette Smoking on Serum Lipid Profile of Males in Nasiriyah City. Medical Journal of Babylon 15, 39-42.

Cobb, C. O., Sahmarani, K., Eissenberg, T. and Shihadeh, A. (2012) Acute toxicant exposure and cardiac autonomic dysfunction from smoking a single narghile waterpipe with tobacco and with a "healthy" tobaccofree alternative. Toxicology Letters 215, 70-75.

Cobb, C. O., Shihadeh, A., Weaver, M. F. and Eissenberg, T. (2011) Waterpipe tobacco smoking and cigarette smoking: a direct comparison of toxicant exposure and subjective effects. Nicotine \& Tobacco Research 13, 78-87.

Diab, O. A., Abdelrahim, E. M. and Esmail, M. (2015) Effect of water pipe tobacco smoking on plasma high sensitivity $\mathrm{C}$ reactive protein level and endothelial function compared to cigarette smoking. The Egyptian Heart Journal 67, 233-241.

Eissenberg, T. and Shihadeh, A. (2009) Waterpipe tobacco and cigarette smoking. American Journal of Preventive Medicine 37, 518-523.

Elias, W., Assy, N., Elias, I., Toledo, T., Yassin, M. and Bowirrat, A. (2012) The detrimental danger of water-pipe (Hookah) transcends the hazardous consequences of general health to the driving behavior. Journal of Translational Medicine 10, 126.

Eliasson, M. (1995) Relationship of cigarette smoking and snuff dipping to plasma fibrinogen, fibrinolytic variables and serum insulin. The Northern Sweden MONICA study. Atherosclerosis 113, 41-53.

El-Zaatari, Z. M., Chami, H. A. and Zaatari, G. S. (2015) Health effects associated with waterpipe smoking. Tobacco Control 24, i31-i43.

Ettehad, D., Emdin, C. A., Kiran, A., Anderson, S. G., Callender, T., Emberson, J., Chalmers, J., Rodgers, A. and Rahimi, K. (2016) Blood pressure lowering for prevention of cardiovascular disease and death: a systematic review and meta-analysis. the Lancet 387, 957-967.

Ghasemi, A., Syedmoradi, L., Momenan, A. A., Zahediasl, S. and Azizi, F. (2010) The influence of cigarette and qalyan (hookah) smoking on serum nitric oxide metabolite concentration. Scandinavian Journal of Clinical and Laboratory Investigation 70, 116-121.

Hakim, F., Hellou, E., Goldbart, A., Katz, R., Bentur, Y. and Bentur, L. (2011) The acute effects of water-pipe smoking on the cardiorespiratory system. Chest $\mathbf{1 3 9}, 775-781$.

Hammal, F., Chappell, A., Wild, T. C., Kindzierski, W., Shihadeh, A., Vanderhoek, A., Huynh, C. K., Plateel, G. and Finegan, B. A. (2015) 'Herbal' but potentially hazardous: an analysis of the constituents and smoke emissions of tobacco-free waterpipe products and the air quality in the cafes where they are served. Tobacco Control 24, 290-297.

Hashem Sezavar, S., Abedi, A. and Sadeghi Bazargani, H. (2004) A comparative study of plasma fibrinogen among hookah smokers, cigarette smokers and non-smokers. Iranian Heart Journal 5, 48-54.

Hawari, F. I., Obeidat, N. A., Ayub, H., Ghonimat, I., Eissenberg, T., Dawahrah, S. and Beano, H. (2013) The acute effects of waterpipe smoking on lung function and exercise capacity in a pilot study of healthy participants. Inhalation Toxicology 25, 492-497.

Islami, F., Pourshams, A., Vedanthan, R., Poustchi, H., Kamangar, F., Golozar, A., Etemadi, A., Khademi, H., Freedman, N. D., Merat, S., Garg, V., Fuster, V., Wakefield, J., Dawsey, S. M., Pharoah, P., Brennan, P., Abnet, C. C., Malekzadeh, R. and Boffetta, P. (2013) Smoking water-pipe, chewing nass and prevalence of heart disease: a crosssectional analysis of baseline data from the Golestan Cohort Study, Iran. Heart 99, 272-278.

Jabbour, S., El-Roueiheb, Z. and Sibai, A. M. (2003) Nargileh (WaterPipe) smoking and incident coronary heart disease: a case-control study. Annals of Epidemiology 13, 570.

Kadhum, M., Jaffery, A., Haq, A., Bacon, J. and Madden, B. (2014) Measuring the acute cardiovascular effects of shisha smoking: a crosssectional study. JRSM Open 5, 205427041453112.

Khan, N. A., Lawyer, G., McDonough, S., Wang, Q., Kassem, N. O., Kas-
Petrus, F., Ye, D., Singh, K. P., Kassem, N. O. and Rahman, I. (2020) Systemic biomarkers of inflammation, oxidative stress and tissue injury and repair among waterpipe, cigarette and dual tobacco smokers Tobacco Control 29, s102-s109.

Koubaa, A., Triki, M., Trabelsi, H., Masmoudi, L., Sahnoun, Z. and Hakim, A. (2015a) Changes in antioxidant defense capability and lipid profile after 12-week low- intensity continuous training in both cigarette and hookah smokers: a follow-up study. PLoS One 10, e0130563.

Koubaa, A., Triki, M., Trabelsi, H., Masmoudi, L., Zeghal, K. N., Sahnoun, Z. and Hakim, A. (2015b) Effect of low-intensity continuous training on lung function and cardiorespiratory fitness in both cigarette and hookah smokers. African Health Sciences 15, 1170-1181.

Layoun, N., Saleh, N., Barbour, B., Awada, S., Rachidi, S., Al-Hajje, A., Bawab, W., Waked, M. and Salameh, P. (2014) Waterpipe effects on pulmonary function and cardiovascular indices: a comparison to cigarette smoking in real life situation. Inhalation Toxicology 26, 620627.

Leone, A. (2003) Relationship between cigarette smoking and other coronary risk factors in atherosclerosis: risk of cardiovascular disease and preventive measures. Current Pharmaceutical Design 9, 2417-2423.

Marshall, M., Ghazipura, M., Hossain, T., Gordon, T. and Chen, L. (2018) Cardiopulmonary risk of waterpipe smoke: a meta-analysis. Journal of Lung Health and Diseases 2, 31-40.

Maziak, W., Taleb, Z. B., Bahelah, R., Islam, F., Jaber, R., Auf, R. and Salloum, R. G. (2015) The global epidemiology of waterpipe smoking. Tobacco Control 24, i3-i12.

Mons, U., Muezzinler, A., Gellert, C., Schottker, B., Abnet, C. C., Bobak, M., de Groot, L., Freedman, N. D., Jansen, E., Kee, F., Kromhout, D., Kuulasmaa, K., Laatikainen, T., O'Doherty, M. G., Bueno-deMesquita, B., Orfanos, P., Peters, A., van der Schouw, Y. T., Wilsgaard, T., Wolk, A., Trichopoulou, A., Boffetta, P., Brenner, H. and on behalf of the CHANCES consortium (2015) Impact of smoking and smoking cessation on cardiovascular events and mortality among older adults: meta-analysis of individual participant data from prospective cohort studies of the CHANCES consortium. BMJ 350, h1551-h1551.

Muddathir, A., Abd Alla, M. and Khabour, O. (2018) Waterpipe Smoking is associated with changes in fibrinogen, FVII, and FVIII levels. Acta Haematologica 140, 159-165.

Nelson, M. D., Rezk-Hanna, M., Rader, F., Mason, O. R., Tang, X., Shidban, S., Rosenberry, R., Benowitz, N. L., Tashkin, D. P., Elashoff, R. M., Lindner, J. R. and Victor, R. G. (2016) Acute effect of hookah smoking on the human coronary microcirculation. The American Journal of Cardiology 117, 1747-1754

Nikolovska Vukadinović, A., Vukadinović, D., Borer, J., Cowie, M., Komajda, M., Lainscak, M., Swedberg, K. and Böhm, M. (2017) Heart rate and its reduction in chronic heart failure and beyond. European Journal of Heart Failure 19, 1230-1241.

Patsopoulos, N. A., Evangelou, E. and Ioannidis, J. P. (2008) Sensitivity of between-study heterogeneity in meta-analysis: proposed metrics and empirical evaluation. International Journal of Epidemiology 37, 11481157.

Pedro-Botet, J., Ascaso, J. F., Blasco, M., Brea, Á., Díaz, Á., HernándezMijares, A, Pintó, X., Millán, J., Grupo de trabajo sobre Dislipidemia Aterogénica and Sociedad Española de Arteriosclerosis. (2020) Triglycerides, HDL cholesterol and atherogenic dyslipidaemia in the 2019 European guidelines for the management of dyslipidaemias. Clínica e Investigación en Arteriosclerosis (in press).

Platt, D. E., Hariri, E., Salameh, P., Helou, M., Sabbah, N., Merhi, M., Chammas, E., Ammar, W., Abchee, A. B. and Zalloua, P. A. (2017) Association of waterpipe smoking with myocardial infarction and determinants of metabolic syndrome among catheterized patients. Inhalation Toxicology 29, 429-434.

Rezk-Hanna, M. and Benowitz, N. L. (2019a) Cardiovascular effects of hookah smoking: potential implications for cardiovascular risk. Nicotine and Tobacco Research 21, 1151-1161.

Rezk-Hanna, M., Mosenifar, Z., Benowitz, N. L., Rader, F., Rashid, M., Davoren, K., Moy, N. B., Doering, L., Robbins, W., Sarna, L., Li, N., Chang, L. C., Elashoff, R. M. and Victor, R. G. (2019b) High car- 
bon monoxide levels from charcoal combustion mask acute endothelial dysfunction induced by hookah (waterpipe) smoking in young adults. Circulation 139, 2215-2224.

Saffar Soflaei, S., Darroudi, S., Tayefi, M., Nosrati Tirkani, A., Moohebati, M., Ebrahimi, M., Esmaily, H., Parizadeh, S. M. R., Heidari-Bakavoli, A. R., Ferns, G. A. and Ghayour-Mobarhan, M. (2018) Hookah smoking is strongly associated with diabetes mellitus, metabolic syndrome and obesity: a population-based study. Diabetology and Metabolic Syndrome 10, 33.

Salahuddin, S., Prabhakaran, D. and Roy, A. (2012) Pathophysiological mechanisms of tobacco-related CVD. Global Heart 7, 113.

Selim, G. M., Elia, R. Z., El Bohey, A. S. and El Meniawy, K. A. (2013a) Effect of shisha vs. cigarette smoking on endothelial function by brachial artery duplex ultrasonography: an observational study. Anadolu Kardiyoloji Dergisi/The Anatolian Journal of Cardiology 13, 759-765.

Selim, G. M., Fouad, H. and Ezzat, S. (2013b) Impact of shisha smoking on the extent of coronary artery disease in patients referred for coronary angiography. Anadolu Kardiyoloji Dergisi/The Anatolian Journal of Cardiology 13, 647-654.

Shafagoj, Y. A. and Mohammed, F. I. (2002) Levels of maximum endexpiratory carbon monoxide and certain cardiovascular parameters following hubble-bubble smoking. Saudi Medical Journal 23, 953-958.

Shafique, K., Mirza, S. S., Mughal, M. K., Arain, Z. I., Khan, N. A., Tareen, M. F. and Ahmad, I. (2012) Water-Pipe smoking and metabolic syndrome: a population-based study. PLoS One 7, e39734.

Shaikh, R. B., Vijayaraghavan, N., Sulaiman, A. S., Kazi, S. and Shafi, M. S. (2008) The acute effects of waterpipe smoking on the cardiovascular and respiratory systems. Journal of Preventive Medicine and Hygiene 49, 101-107.

Shihadeh, A. (2003) Investigation of mainstream smoke aerosol of the argileh water pipe. Food and Chemical Toxicology 41, 143-152.

Shishani, K., Howell, D., McPherson, S. and Roll, J. (2014) Young adult waterpipe smokers: Smoking behaviors and associated subjective and physiological effects. Addictive Behaviors 39, 1113-1119.

Sibai, A. M., Tohme, R. A., Almedawar, M. M., Itani, T., Yassine, S. I., Nohra, E. A. and Isma'eel, H. A. (2014) Lifetime cumulative exposure to waterpipe smoking is associated with coronary artery disease. Atherosclerosis 234, 454-460.

Sterne, J. A., Hernán, M. A., Reeves, B. C., Savović, J., Berkman, N. D., Viswanathan, M., Henry, D., Altman, D. G., Ansari, M. T., Boutron, I., et al. (2016) ROBINS-I: a tool for assessing risk of bias in nonrandomised studies of interventions. BMJ 355, i4919.

Vigo, D. E., Pérez Lloret, S., Videla, A. J., Pérez Chada, D., Hünicken, H. M., Mercuri, J., Romero, R., Nicola Siri, L. C. and Cardinali, D. P. (2010) Heart rate nonlinear dynamics during sudden hypoxia at $8230 \mathrm{~m}$ simulated altitude. Wilderness and Environmental Medicine 21, 4-10.

Ward, K. D., Ahn, S., Mzayek, F., Al Ali, R., Rastam, S., Asfar, T., Fouad, F. and Maziak, W. (2015) The relationship between waterpipe smoking and body weight: population-based findings from Syria. Nicotine and Tobacco Research 17, 34-40.

Waziry, R., Jawad, M., Ballout, R. A., Al Akel, M. and Akl, E. A. (2018) The effects of waterpipe tobacco smoking on health outcomes: an updated systematic review and meta-analysis. International Journal of Epidemiology 46, 32-43.

Wolfram, R. M., Chehne, F., Oguogho, A. and Sinzinger, H. (2003) Narghile (water pipe) smoking influences platelet function and (iso)eicosanoids. Life Sciences 74, 47-53.

Wu, F., Chen, Y., Parvez, F., Segers, S., Argos, M., Islam, T., Ahmed, A., Rakibuz-Zaman, M., Hasan, R., Sarwar, G. and Ahsan, H. (2013) A prospective study of tobacco smoking and mortality in bangladesh. PLoS One 8, e58516. 